\title{
eHealth Turning Points as Forced by the Covid-19 Dramatic Experience
}

\author{
Francesco PINCIROLI \\ Dipartimento di Elettronica, Informazione e Bioingegneria - Politecnico di Milano
}

\begin{abstract}
In this ongoing fall of the year 2021, many disciplines are frightened by the Covid-19 situation. A generalized sense of Scientific and administrative impotence, - in keeping the pandemic under real control, - is felt widely in Society. In this Invited Lecture the author reminds us of the blows suffered, recalls pertinent elements present in our social organization, browses selected eHealth experiences and proposes an open agenda of actions to allow the eHealth to help the population segments better, and individuals as well.
\end{abstract}

Keywords eHealth, pHealth, Covid-19

\section{Foreword}

In appreciating and accepting the opportunity of an Invited Lecture at pHealth2021, I take the liberty, and the responsibility as well, of sharing with the audience of our community the major professional eHealth reflections that have come to my mind during the Covid-19 not-yet-concluded events. My default point of view in living in such a period is that of a "senior citizen and retired Professor of Bioengineering and eHealth", who spent his entire academic career in the field [1-8]. I take this liberty in the hope of being of some help in letting our Community focus and discuss possible turning points to be considered while continuing to attempt to understand, develop and design new directions that our field will take, or even be forced to take. In putting my liberty into practice, I believe that my main - and more or less only - reference is my long-lasting academic background. Therefore, my expected result is a document to be placed on our community table for discussion. We need this discussion to be rapid, otherwise we will just be overwhelmed by the development of events largely external to us.

\section{The Blows}

I perceived some of the events related to the Covid-19 situation as real blows. Here they are.

In the multi-steps eHealth pipeline from data to knowledge, our Covid-19 professional drama started with so many people dying rapidly, in so many different Countries, with no credible data, and no time to verify the number of deaths. The footage of rows of military trucks transporting the coffins of the deceased - at night to an

\footnotetext{
${ }^{1}$ Corresponding Author: Francesco Pinciroli FIAHSI, Prof. Emeritus, Dipartimento di Elettronica, Informazione e Bioingegneria - Politecnico di Milano, Milano, Italy Email: francesco.pinciroli@polimi.it
} 
unknown location from the medium-size Italian city of Bergamo, in the Lombardy region, since there were not sufficient resources to cremate them: these powerful images shown on the TV News hit my mind and constricted my heart. To me, this scene also included the perception of failure in engineering, a failure where the much-appreciated ICT for Healthcare gave zero-help. The high transmission speeds reached by our ICT infrastructures over recent decades were unable to provide effectiveness. We were not able to give help in a situation of contagion. This blow to my engineering background was even greater than the one I had had a few months earlier, when on August 14, 2018, the Morandi Bridge, the main highway bridge in the center of Genoa, collapsed.

We have in our history the historical success of about 50 years of eHealth: from Medical Informatics helping statistics, to ICT for health, even adding fantastic just-intime skills, allowing people to be saved in the event of sudden heart attacks. Later, we started to do Data Mining, sustained by the large abundance of data produced free-ofcharge. Then came eHealth, which includes easy-to-be-immediately reshaped display facilities. All of this was Science. But the Covid-19 period started without data, apart from those counted for infected, dead and hospitalized subjects. If data are not generated, we do not have any eHealth segment to play with. Without data, our Scientific Community is outside the circle helping our Society.

Immediately after the emergence of Covid-19, from month to month we have seen virologists on TV addressing the TV audience with messages meant to be or be perceived as different from one another, even if none of them was fake. Despite our attitude to label the eHealth approach "comprehensive", the methods and the technologies we are familiar with have not proved helpful in letting the audience, and the virologists, come up with better truths themselves.

\section{Remarks about our Social Organization}

During the Covid-19 period I matured and/or renewed some obvious observations, that I consider pertinent. They do not have the sudden power of a slap. Nevertheless, they can be even more relevant in the process of building the future of our Community. Here they are.

A judge, a priest, a game player, a leader, a physician: these were necessary roles made and identified since the beginning of mankind. However, scientists, and even engineers came later, after some tools had become available, tools to be used in the management of appropriately selected and significant data and materials, and to obtain results that can be repeated. So, as scientists, we are late comers in the Society, while Covid-19 is an ancestral player in Society's evolution. Nevertheless, Society believes in us because we do not change the knowledge we manage; once released, it is stable. It does not include mistakes.

We have to recognize and accept the substantial, intrinsic inability of scientists to manage the population governance at a national level. I mean that all of us, engineers and related disciplines, have a background that grew up under the umbrella of those experimental approaches where somebody else is allowed to repeat your experiments, and he/she is supposed to obtain the same results. Instead, the population governance is requested to be, at any level, the capacity of finding an ongoing baricenter among inhomogeneous needs and/or desires, and to do this, keep society in quiet relationships between citizens. So, to be a governor, a significantly different background is required from the scientific background. Having recognized this, our eHealth Community holds 
the role to continue to do eHealth, in support of the Country's governance. The Covid19 event also triggered rapid changes in yesterday priorities, to tackle the lack of knowledge and to provide practices for the need for governance.

Although each of us is a scientist, none of us is able to persist in behaving fully as a scientist when he/she is in front of the TV. Here we are just citizens, exposed in full to the technicalities of the communication-for-persuasion arena. I think about a car advertisement on TV where, for years, they did not include any information about speed, acceleration, brakes, gas consumption, and other historically technical quantities. Instead, they outline the emotion that the car generates in the owner's soul. And, even worse, the advertisement on TV for hearing aids just says that they cannot be seen by anyone you meet. Not a single technical detail, about how and why our auditory functions might be improved, is forwarded to the audience.

Still, in recent years, my experience in doing home eHealth saw a hospital-dependent variety of accreditation modalities. The public hospitals in my Region attempt to follow a common modality. But each private hospital has its own way. This occurs even if it has been accredited, i.e., verified by the Regional Healthcare Authority as being able to provide the citizen with the same level of care as a public hospital. So, in my role of citizen/patient, for clinical examinations, I choose public or private according to the shortness of the waiting lists. If I wish to put together all the clinical data I have generated in different places, I have to enter each hospital website with its/my credentials. Clinical and healthcare databases are not aligned. At my age, my accreditation modalities occupy about an entire page of my agenda. I do the grouping on my PC. I never received alerts - not from any public or private hospitals, nor by the Regional Healthcare Information System - about any bad - and even hacking - maneuvers attempted on the websites where some of my healthcare data are resident.

\section{Browsing on eHealth Experiences}

In the middle of September 2021, Italian newspapers published the new Laws on the "Green Pass", since this Covid-19 document is a top subject. Its eventual mandatory level and the society segments to be submitted to such a policy are under a wide political and social debate. Nevertheless, under an eHealth point of view, that focuses on how to collect the data only to release it to any citizen, and how to deliver it to the recipient, the "Green Pass" is not a problem. Let me just mention that all the members of my family have it, including my grandchildren, whose ages range from 21 to 12. I easily downloaded my "Green Pass" after having received an SMS including the instructions. This message arrived after I had had my second injection of the vaccine. The eHealth procedure is extremely simple and effective. We are in front of an eHealth case of success.

We can go back 50 years, in order to start helping Statisticians again to do their jobs better on populations, to compare sub-groups of populations, to foresee evolutions, etcetera. Similarly, we can start again in helping physicists to plan their instruments better to detect, identify and rank this new Covid-19 virus and its variants too. Within Pulmonary Intensive Care Units, we can empower the storing capabilities for bio signals. In doing so we facilitate the pointing out of a better taxonomy of what Covid-19 does on our pulmonary system. But this is for the future. At present people are dying, and dying fast, with low connections to the contents of the hypothesized "Personalized Life-Long Healthcare and Clinical Record" our eHealth was "almost-ready" to provide. 
For some years, our eHealth Community has had to worry about the wide spread of Fake News. Even worse for my mentality, I had to learn from psychologists that, in navigating healthcare information on the Internet, citizens are inclined to follow a personally sustainable and step-by-step pathway towards the capture of elements capable of supporting their prejudices. So, by doing eHealth, it is not enough to produce new knowledge and to make it easily available to potential recipients. We must also be concerned about convincing such recipients that the knowledge we offer is a good one, eventually providing such persons with methods and tools for unmasking fakes. And what our community would do when faced with citizens highly inclined to give value to sources that only resonates with their inclinations/prejudices? Can "Blah ... this is not a matter for eHealth!" be an answer? Probably not, as it can devalue much of the comprehensive eHealth approaches and results. We are facing the persisting absence of suitable and instant-to-be-used confidence indicators for data sources, tuned to the basic understanding of the envisaged users.

Historically, as citizens, when looking for clinical and healthcare information, we use "physical signs". For instance, when at the newspaper shop I buy the "weekly healthcare focus" added to many newspapers, I foresee that I'll read it easily. I won't have any problems in understanding what I read. However, what I read will be nothing more than a basic generic introduction, even when I find myself interested in the title of the article. Normally, something opposite occurs when, as a citizen, I enter the PubMed website, where I can do queries based on the scientific and specific name of my symptoms and diseases. This behavior of mine will lead me to select scientifically valuable and focused information sources, but I do not have the sufficient background knowledge to understand what I read. Unfortunately, by entering the generic Internet arena, we do not have significant symbols available, even if something starts to exist. For instance, as a citizen, I believe that the websites of some well-known and credited hospitals provide a reasonably trustworthy symbols. Of course, they must be tuned to the asymmetric medical lexicons belonging to specific segments of the population.

The main gate in my courtyard, in the central district of Milan, overlooks a narrow sidewalk. When I exit through the gate, I look left and right. I do so "normally", with the expectation that the sidewalk could be occupied by pedestrians. My body's sensor and actuator systems are tuned to the expected speed range of a human walking. For a couple of years I have had to change this assumption of mine. I started doing so from the moment when, on exiting the gate, I was lucky not to be hit by an electrical scooter running on the sidewalk. This fact was for me a "lesson learned", from a sudden experience, fortunately for free. Such racing was not (not yet?) illegal, because we were at the very beginning of the emergence of the electrical scooter market. In other sudden cases or situations, the "lessons learned" can be costly, even physically. And the sudden Covid19 situation has changed our best practices, with such fast timing that we know it is impossible to be adopted by our eHealth Standardization Bodies. This makes the discovery of new Best Practices in eHealth more important than what it usually is.

In recent years, the experience doing home banking has seen the methods of accreditation widen, up to the current way consisting usually of username + password + OTP code, the latter received on the smartphone is only valid for a few minutes. In truth, the expansion came from something bad which occurred while using earlier more limited methods. Regarding these bad things which, in the end, happened to someone else, my bank never told me any details. Nevertheless, they sent me warnings such as "Never update your credentials by answering an email and using the included links! We never use this modality!". Moreover, they often add "please carefully verify, letter by letter, 
our exact name as the correct sender of an email to you." And, when I receive something strange, I take a picture of it on the PC screen and I send it to the contact person linked to my bank account. Of course, I only do this action from within my section on the bank's website. To reach my section, I would have had to use my updated credentials in full. Subsequently, usually, the contact person sends me a reply, within the protected area, where they thank me for having alerted them. Let me label what I have just described as "Lessons learned from the user by interacting over time with system/platform management". Both progressive and interactive accreditation modalities should be recommended during eHealth contacts between the patient and healthcare platforms.

Obviously, I share the sense of deep sadness in the face of the many millions of deaths by Covid-19. However, given the audience that we are, let me synthetize that worse was caused by a lack of knowledge. At present we see that every Country is trying to amend the situation by uncovering some lines of reactions. For sure they ask for investments, that the governments of our Countries envisage as "recovery funds". A generally accepted line along which to use such money is "Innovation", which is widely recognized as including the "digital transformation". The eHealth infrastructures do not show any difficulty in being recognized as part of the "digital transformation". In addition, eHealth and Telemedicine confirm the promise of getting "things done even at a distance", helping to counteract the close contacts still necessary during the Covid-19 contagion. So, it seems certain that eHealth and Telemedicine can count on a significant increase of funds available for their programs. It matters little that, despite the efforts made in the recent decades, Telemedicine did not exceed the threshold to become widely accepted in everyday practice. With Covid-19, the modality of acting from a distance has emerged as extremely necessary. It is a life-saving necessity. It is no longer a cost/effectiveness approach, as it was before.

There are reasons why eHealth never considered the general practitioner's office as a significant and structured source of data [7]. In such an environment few standards are used, and the generated data are not a lot. Furthermore, the information generated by patient-physician interactions often belongs to conversational language. The evasion of "sending the patient to a specialist" is an open door for GP behavior, sometimes also due to elements generically belonging to the area of Defensive Medicine. But the Covid-19 situation does not leave this scenario unchanged. The awareness of the relevance of the timely recognition of emerging symptoms and the alarming risk of the patient causing a risky contagion, even within his/her family, are the major changes. Also, because, probably in every Country, the current dimension of the hospital departments proved largely insufficient for Covid-19 patient populations. In front of them, eHealth will never be able to decrease the chances of contagion by taking food to the door of the apartment where a patient is obeying his/her quarantine duties. Nevertheless, eHealth can think about actions dedicated to the GP outpatient environment with more conviction than what has been done in the past, also counting - since this is new and facilitating, - on high attention and interest from the government.

As happens in any significantly established segment of the knowledge, and even the market, our community has generated a certain number of associations over the decades. Each of them is now faced with what is generated by the Covid-19 situation. The activities of some of them are published on their websites, usually under a branch that has a name that includes the term Covid-19. The associations do so unavoidably bearing in mind their own survival, in order to not be overwhelmed by the generalized perception of the weaknesses of their own tools and infrastructures in the face of so many deaths. The high number of scientific publications found by PubMed when we do an "eHealth + 
Covid-19" query is certainly significant. [By September 15, 2021, PubMed counts some 6365 results.] But all such activities come from the attempt to extend to the appearance of Covid-19 the research approaches already designed before it, when those researches were conceived, i.e. well before Covid-19. Our community must promote the design of entirely-new research.

The inclination of simply adding "Covid-19 oriented" to previously conceived and existing programs and approaches does not ignore commercial products [9]. In reality, the availability of "recovery fund" resources may exacerbate the situation. If this happens "too much", we will shortly face the risks of losing both government money and our credibility. We have to avoid this happening. We have to be the first and effective referees of the appropriateness of the emerging eHealth products said to be of help in the Covid-19 pandemic.

Due to the Covid-19 situation, we need to anticipate changes in the curricular education. Let me think about the Biomedical Engineering pathway, taking into account the four historical pillars of Mechanics, Electronics, Chemistry and Information Science. I believe that the students' educational paths would be strengthened in Biostatistics, should include basics notions in Epidemiology and in Clinical Laboratory, and Lab-ona-Chip machines. Other seminar additions to include would be Patient Rights and Healthcare Data Protection, basic Psychology of the Internet, users of Clinical and Healthcare Information Sources. Research on Instant Scoring Tools of Information Sources and on Instant Data Science would also be promoted. Symmetrically, for the curricula in Medicine and Life Sciences, we should become able to define the contents to offer distance seminar courses in "Instant eHealth".

\section{Discussion}

As after any war, we respect the dead and we have to continue to work hard for the care of the survivors, particularly when some of them still need to be treated for diseases other than Covid-19. eHealth is a mature adult in its 50's, i.e., it is an experienced entity, that still has a lot of power to single out innovation and to keep it tuned to the real needs of the entire population [10]. So, our Community will continue to work hard. Our role as "late comers" awaits the additionally new and robust scientific knowledge that we will help to discover.

A question which we will never know the exact answer to is: how many more deaths would have occurred in the current not-yet-ended pandemic period if we had not had the normally running ICT-for Health infrastructures available, even if we say every day that they should work better?

\section{Conclusions}

According to what has been comprehensively caused in healthcare by Covid-19, there are some lines of development that we need to start caring about more than in the past [9]. Given my foreword and after the reflections I have shared with you here above, I endeavor to suggest a possible open agenda of courses of action.

Find indicators and/or "markers" of trust for healthcare and clinical information sources that can be used "instantly" by users, including "rapid tests" that the user can do on the source to establish whether he/she can believe it or not. 
Take the Standardization Bodies involved in setting up procedures intended for "rapid best practices"

Revise the contents of the educational pathways of any level, centered on and/or including eHealth.

The General Practitioner office should be considered, studied and helped more than in the past.

We as individuals, as well as through our eHealth Associations, have to remain as close as we can to the Healthcare Government Offices and Officials, including the level of Ministries, especially in the role of eHealth auditors, and also informed promoters.

We have to act by staying intelligently alert and by being respectful professionals, skilled designers and rigorous scientists.

\section{References}

[1] Pinciroli F, Anderson J (ed.) Changes in Healthcare Instrumentation due to Microprocessor Technology. Amsterdam: North Holland; 1981, pp 326

[2] Meester GT, Pinciroli F (ed.) Databases for Cardiology. Dordrecht: Kluwer Academic Publishers; 1991, pp. 418

[3] Pinciroli F (ed.) Informatica di Base per la Medicina. Torino: UTET; 1992,pp 661

[4] Cristiani P, Pinciroli F, Stefanelli M (ed.) I Sistemi informativi Sanitari. Bologna: Pàtron Editore; 1996, pp 300

[5] Pinciroli F, Masseroli M (ed.) Elementi di Informatica BioMedica. Milano: PoliPress; 2005, pp 382

[6] Pinciroli F, Bonacina S (ed.) Applicazioni di Sanità Digitale. Milano: PoliPress; 2009, pp 390

[7] Marceglia S., Ferrante S., Bonacina S., Pinciroli F., Lasorsa I., Savino C., Pozzi G., Domains of Health IT and Tailoring of Evaluation: Practicing Process Modeling for Multi-Stakeholder Benefits. Stud Health Technol Inform. 2016; 222: 63-76

[8] Quaglini S, Cesarelli M, Giacomini M, Pinciroli F (ed.) eHealth-Medicina Digitale. Bologna: Pàtron Editore; 2017, pp 362

[9] Scott RE, Mars M. Editorial Covid-19 and eHealth: A Promise of Peril Paradox?, J Int Soc Telemed eHealth 2021;9:e1

[10] Demiris G (ed.) E-Health: Current Status and Future Trends. Stud Health Technol Inform. 2004; 106, pp160 